\title{
Trabalho voluntário: uma alternativa para a promoção da saúde de idosos
}

\author{
VOLUNTARY WORK: AN ALTERNATIVE TO PROMOTE HEALTH FOR THE ELDERLY \\ TRABAJO VOLUNTARIO: UNAALTERNATIVA PARA LA PROMOCIÓN \\ DE LA SALUD DE ADULTOS MAYORES
}

Luccas Melo de Souza', Liana Lautert ${ }^{2}$

\section{RESUMO}

Trata-se de uma reflexão temática que objetiva apresentar o trabalho volun-tário como uma alternativa para a promo-ção da saúde dos idosos brasileiros. Par-tindo da definição do trabalho voluntário, o artigo contextualiza tal atividade no cenário brasileiro, expondo-o, também, como um instrumento para 0 alcance de um enveIhecimento ativo e saudável. Apresenta estudos internacionais com idosos que realizam voluntariado, os quais encontraram relação entre o trabalho voluntário e satisfação pela vida, menos sintomas depressivos e avaliação positiva da vida, quando comparados com idosos que não o realizam. Concluiu-se, reforçando a importância dos profissionais de saúde a estimularem e a facilitarem o acesso dos idosos à realização do trabalho voluntário, considerando esta atitude um desafio criativo e inovador para a promoção da saúde dos idosos.

\section{DESCRITORES}

Saúde do idoso.

Promoção da saúde.

Envelhecimento.

Trabalhadores voluntários.

\begin{abstract}
This article is a thematic reflection. It aims at presenting volunteer work as an alternative for promoting health for elders in Brazil. Having as a starting point the definition of volunteer work, this study contextualizes this type of activity in Brazilian society, explaining it also as an instrument that makes possible for people to experience an active and healthy aging process. The text presents international studies with elders who volunteer and, as a result, have found a relationship between volunteering and satisfaction with their lives and have had less symptoms of depression and a more positive evaluation of life when compared to elderly who do not volunteer. The conclusion emphasizes the importance for health professionals to stimulate and facilitate the access of elders to volunteer work, considering this posture a creative and innovative challenge for the promotion of health for the elderly.
\end{abstract}

\section{KEY WORDS}

Health of the elderly.

Health promotion.

Aging.

Voluntary workers.

\section{RESUMEN}

Este artículo trata de una reflexión temática que tiene como objetivo presentar el trabajo voluntario como una alternativa para la promoción de la salud de los adultos mayores brasileños. Partiendo de la definición del trabajo voluntario, el artículo contextualiza tal actividad en el escenario brasileño, exponiéndolo, también, como un instrumento para alcanzar un envejecimiento activo y saludable. Presenta estudios internacionales con adultos mayores que realizan voluntariado, los cuales encontraron relación entre el trabajo voluntario y satisfacción por la vida, menos síntomas depresivos y evaluación positiva de la vida, cuando fueron comparados con adultos mayores que no lo realizaban. Se concluye, reforzando la importancia de que los profesionales de la salud estimulen y faciliten el acceso de los adultos mayores a la realización del trabajo voluntario, considerando esta actitud un reto creativo e innovador para la promoción de la salud de los adultos mayores.

\section{DESCRIPTORES}

Salud del anciano.

Promoción de la salud.

Envejecimiento.

Trabajadores voluntarios.

${ }^{1}$ Enfermeiro. Mestrando do Programa de Pós-Graduação em Enfermagem da Universidade Federal do Rio Grande do Sul (UFRGS). Bolsista CAPES. Porto Alegre, Rs, Brasil. luccasms@gmail.com ²Enfermeira. Doutora em Psicologia. Professora Adjunta do Programa de Pós-Graduação em Enfermagem da UFRGS. Porto Alegre, RS, Brasil. lila@enf.ufrgs.br 


\section{INTRODUÇÃO}

0 trabalho voluntário é uma forma de participação social que vem em constante crescimento à medida que transcorre o tempo. Tamanho é esse aumento que, a fim de estimular ainda mais essa atividade, 0 ano de 2001 foi escolhido, pela Organização das Nações Unidas (ONU), como o Ano Internacional do Voluntariado. Entretanto, não se pode associar 0 avanço do voluntariado somente a essa iniciativa da ONU.

No Brasil e na própria ONU, outros documentos foram publicados, posteriormente, com a finalidade de despertar a participação das pessoas em atividades voluntárias - principalmente os jovens e os idosos -, no intuito de investirem seu tempo livre na promoção da solidariedade e da cidadania.

Destaca-se que, entre os idosos - particularmente os aposentados - o voluntariado é uma prática comum e em crescente expansão, servindo como mecanismo para manterem-se socialmente ativos e afastarem-se do preconceito advindo com a aposentadoria, entre outros benefícios. Contudo, no Brasil pouco se sabe a respeito da influência do voluntariado na saúde dos idosos, pois ainda há carência de pesquisas a respeito dessa relação. 0 grande número de publicações brasileiras sobre esse tema é oriundo de áreas como a psicologia e serviço social, que são as pioneiras na realização de estudos envolvendo o trabalho voluntário.

A ONU(1) e a Organização M undial de Saú$\mathrm{de}^{(2)}$, por sua vez, têm alertado para a necessidade premente de pesquisas voltadas a esse tema, na intenção de estimular o trabalho voluntário, considerado uma ferramenta para 0 alcance de um envelhecimento ativo e saudável.

Nos Estados Unidos, nos países da Europa e até mesmo da Ásia, por exemplo, essa temática tem sido debatida há algumas décadas, sendo, inclusive, alvo de estudos de enfermeiros e médicos, diferentemente da realidade brasileira. Esses países, acreditando que o trabalho voluntário sirva como uma estratégia para a promoção da saúde, especialmente dos idosos - reforçando os pressupostos da Teoria da Atividade -, têm investido em pesquisas sobre os reflexos do voluntariado na saúde e na qualidade de vida das pessoas.

Nesse contexto, esse artigo tem o objetivo de apresentar o trabalho voluntário como uma alternativa para a promoção da saúde dos idosos brasileiros e como um instrumento para alcançarem um envelhecimento ativo. Para isso, organiza uma reflexão temática construída a partir de livros, legislações, dissertações, teses e artigos encontrados no Portal de Periódico CAPES, nas bases de dados LILACS e PUBM ED, na biblioteca eletrônica SCIELO e no Sistema de Automação de Bibliotecas da Universidade Federal do Rio Grande do Sul.

Foram pesquisados artigos que compreendiam os últimos dez anos (entre os anos de 1996 e 2006). A esses, foi incluído um estudo ${ }^{(3)}$ realizado com voluntários de Minessota no ano de 1991, por considerar-se esse uma referência na temática. Para a busca bibliográfica, foram utilizados os termos idoso (aged), envelhecimento (aging), trabalhadores voluntários (voluntary workers), voluntariado (volunteering) e promoção da saúde (health promotion). Subseqüente a essa busca, efetuou-se a leitura exploratória do material, obtendo-se uma visão integral desse. Em seguida, realizou-se a leitura seletiva, momento em que se definiu quais materiais realmente compreendiam a temática desse estudo. Os materiais selecionados foram organizados consoante 0 interesse da argumentação pretendida.

\section{DEFININDO E CONTEXTUALIZANDO O TRABALHO VOLUNTÁRIO}

Apresentar uma definição exata que descreva totalmente o trabalho voluntário não é uma tarefa fácil, pois do mesmo modo que o trabalho remunerado, o voluntariado abrange uma complexa esfera de atividades. Todavia, diferentemente do primeiro, 0 trabalho voluntário não possui um sistema de classificação padronizado que oriente sua categorização e definição(3). Apesar dessa dificuldade, pode-se definir o trabalho voluntário como sendo qualquer atividade onde a pessoa oferta, livremente, o seu tempo para beneficiar outras pessoas, grupos ou organizações, sem retribuição monetária(4-5).

Pelos conceitos de trabalho voluntário utilizados na maioria dos estudos sobre o tema, é possível destacar algumas convergências, entre eles: as ações estão direcionadas às comunidades ou às pessoas mais carentes do que o voluntário, onde está implícita a solidariedade. Outro ponto em comum é a doação do tempo livre, o que supõe a sua gratuidade, pois sua realização não envolve a remuneração financeira(6).

Convém destacar que o trabalho voluntário pode compreender uma faixa estreita ou ampla de ações, podendo incluir desde tarefas efetuadas para/com organizações trabalho voluntário formal - como também ajuda a um vizinho ou familiar - trabalho voluntário informal ${ }^{(3)}$. 0 voluntário é aquele indivíduo que se oferece para prestar um serviço, por vontade própria, a partir de suas inquietações pelos problemas sociais, sem receber remuneração econômica para isso(7). 
No Brasil, o trabalho voluntário é legislado pelo art. 1 o da Lei № 9.608, de 18 de fevereiro de 1998, a qual o considera como a

atividade não remunerada, prestada por pessoa física a entidade pública de qualquer natureza, ou a instituição privada de fins não lucrativos, que tenha objetivos cívicos, culturais, educacionais, científicos, recreativos ou de assistência social, inclusive mutualidade ${ }^{(8)}$.

Para se compreender o desenvolvimento dessa atividade no Brasil, é preciso conhecer um pouco da sua origem e vinculação. Sua história está atrelada a fundação da Santa Casa de Misericórdia em Santos, no ano de 1532, fator que influenciou seu foco voltado à assistência social e, principalmente, à religião(9).

Decorrente desse vínculo com a religião, o modelo do voluntariado foi firmado e difundido embasado nos preceitos religiosos, sendo desde o seu início associado à caridade e ao humanitarismo. Ressalta-se, da mesma forma, o caráter filantrópico do voluntariado como uma forte marca histórica herdada dos religiosos, sendo até os dias de hoje um componente presente tanto na motivação quanto nos objetivos das instituições que o desenvolvem ${ }^{(6)}$.

Atualmente, o voluntariado está fortemente relacionado, também, com as Organizações Não-Governamentais (ONGs). Essas,são instituições privadas que não possuem fins lucrativos, uma vez que os eventuais lucros são redirecionados para os objetivos e para as atividadesfim da organização, não podendo ser distribuídos entre os seus integrantes ${ }^{(6)}$.

Outros grupos que vêm organizando-se e apresentando o trabalho voluntário como alternativa criativa e concreta para aproveitar o potencial dos idosos são as Universidades da Terceira Idade. Algumas dessas estimulam e propõem, ao idoso, o voluntariado por meio da realização de atividades culturais, físicas, artísticas e sociais, de acordo com suas habilidades, desejos e afinidades ${ }^{(10)}$. Com esses incentivos, muitos indivíduos encontram a possibilidade de manterem-se ativos, principalmente após o momento da aposentadoria, preenchendo o tempo disponível auxiliando outras pessoas. Assim sendo, considerase o trabalho voluntário como uma forma de ajuda mútua, onde os idosos que o realizam assessoram a outras pessoas ao mesmo tempo em que se sentem úteis e inseridos na sociedade, refletindo - essa troca -, também, na saúde e na qualidade de vida dos voluntários.

\section{TRABALHO VOLUNTÁRIO: FERRAMENTA PARA O ENVELHECIMENTO ATIVO}

No último século, o fenômeno de transição demo-gráfica, caracterizado pelo aumento rápido do número de idosos na população - com início nos países desenvolvidos e que hoje alcançou, também, os países em desenvolvimento - trouxe consigo várias mudanças na socieda- $\mathrm{de}^{(11)}$. Decorrente disso, levantaram-se diversas interrogações fundamentais e novas demandas para os responsáveis pela elaboração de políticas públicas, a fim de suprir as necessidades e atender a uma população com percentual crescente de pessoas idosas.

Para que se enfrente a realidade de uma população envelhecida, soluções criativas e viáveis devem ser adotas pelos países, especialmente aqueles em estágio de desenvolvimento. Salienta-se que o envelhecimento da população tem exigido respostas no que tange a formulação de políticas públicas de saúde e políticas sociais dirigidas aos idosos, na intenção de preservar a saúde destas pessoas e melhorar sua qualidade de vida, dividindo, então, as atenções e conduzindo-as para além do tradicional atendimento às doenças e seus agravos ${ }^{(12)}$.

Nesse sentido, a ONU e a OM S têm externado sua preocupação em atender à população de idosos, estabelecendo novas opções de políticas públicas e incentivando os países para que as instituam. Uma das alternativas criativas estimuladas por esses órgãos diz respeito ao envolvimento dos idosos com o trabalho voluntário.

A ONU, através das propostas do Plano de Ação Internacional para o Envelhecimento, coloca a participação dos idosos na sociedade, através da realização do trabaIho voluntário, como um dos objetivos e compromissos a serem adotados pelos países preocupados em manter uma sociedade para todas as idades. Esse documento reconhece que a contribuição social dos idosos vai além de suas atividades econômicas, pois muitas de suas valiosas colaborações não se medem nos termos econômicos, como nos cuidados prestados aos membros da família e na realização de trabalhos voluntários na comunidade, servindo, estas e outras ações, para aumentar e manter 0 bem-estar pessoal e coletivo ${ }^{(1)}$.

Contudo, para isso, é preciso oferecer oportunidades e programas de apoio para estimular os idosos a participarem de atividades tais como o trabalho voluntário. Há que se abrir espaços para a socialização dos idosos, através da criação de ambientes que possibilitem a prestação de serviços voluntários em todas as idades, reconhecendo 0 valor público dessa atividade e facilitando a participação dos idosos ${ }^{(1,13)}$.

Nessa mesma linha de pensamento, a OM S considera o voluntariado como elemento importante para a manutenção do bem-estar e da qualidade de vida na velhice, além de ser uma proposta para o envelhecimento ativo. Esse é o processo de otimização das oportunidades de saúde, participação e segurança, cujo objetivo é o de manter a qualidade de vida à medida que as pessoas ficam mais velhas. Esse conceito é aplicável tanto a indivíduos quanto a grupos populacionais, destacando-se que a palavra ativo refere-se à participação contínua nas questões sociais, econômicas, culturais, espirituais e civis, e não somente ao fato de estar fisicamente ativo ou de participar da força de trabalho(2), pois se sabe que a qualida- 
de de vida na velhice não depende somente de condições físicas e/ou biológicas, uma vez que fatores sociais, psicológicos e ambientais também são relevantes e determinantes para 0 bem estar ${ }^{(14)}$.

Acredita-se que a proposta do envelhecimento ativo sirva como base para a elaboração de políticas públicas multisetoriais de envelhecimento, a fim de melhorar as condições de saúde e aumentar a participação entre as populações que estão envelhecendo. Entretanto, admitese que isso não é fácil de se conseguir, ao passo que exige tempo e esforço dos envolvidos ${ }^{(2)}$.

Para que se atinja a meta do envelhecimento ativo, as autoridades, as ONGs, o setor privado e os profissionais do serviço social e da saúde devem, primeiramente, ajudar a promover redes de contatos sociais para as pessoas idosas, no propósito de minimizar o isolamento social que muitas destas pessoas estão sujeitas. Deve-se preparar e adaptar os idosos para que a realidade social que estamos perpassando não limite sua participação social. Há que se desenvolver alternativas de organização social e comunitária que, contrariamente à situação atual, permitam ao idoso continuar sendo parte da estrutura social, participando ativamente nela ${ }^{(2,13)}$. Nesse sentido, acredita-se no trabalho voluntário como uma dessas redes sociais, servindo com um mecanismo de ajuda para romper esse afastamento social a que muitos estão submetidos.

Dessa forma, oferecer oportunidades para o desenvolvimento de programas que envolvam o voluntariado entre idosos é um desafio a ser enfrentado por um país como o Brasil, ao passo que muitos idosos são afastados do mercado de trabalho e da vida social, além de serem relativamente impedidos de terem um projeto de vida ${ }^{(7)}$.

Assim, salienta-se que é o momento de termosum novo paradigma, transpondo a tendência atual - que julga a velhice como um fardo social - para uma concepção que considere o idoso como participante ativo de uma sociedade com integração social, contribuinte ativo e beneficiário do seu desenvolvimento(2,13,15).

Desse modo, na perspectiva do envelhecimento ativo e para que a velhice seja uma fase de experiências positivas, a vida deve ser acompanhada de oportunidades contínuas que permitam promover a saúde através, inclusive, da participação social dos idosos. Esses, a cada ano que passa, aumentam em número e em proporção, adquirindo idades mais avançadas com melhor saúde e maior independência, necessitando, assim, de alternativas que lhe acrescentem prazer e satisfação para viverem e desfrutarem os anos conquistados.

Reforça-se que saber aproveitar o potencial do idoso por meio do trabalho voluntário é, também, uma ferra- menta para retirá-lo da exclusão social, devolvendo-lhe a auto-estima e ajudando-o a exercer sabiamente a sua cidadania $^{(7)}$

\section{TRABALHO VOLUNTÁRIO: ALTERNATIVA PARA A PROMOÇÃO DA SAÚDE DE IDOSOS}

O conceito moderno e a prática de Promoção da Saúde surgiram e desenvolveram-se mais vigorosamente nos últimos 20 anos, nos países desenvolvidos, particularmente no Canadá, nos Estados Unidos e nos países da Europa Ocidental|(16). A primeira grande Conferência Internacional sobre a Promoção da Saúde foi realizada em Ottawa, em 1986, da qual resultou a conhecida Carta de Ottawa, principal marco de referência para a Saúde Pública no mundo ${ }^{(17)}$.

No presente artigo utiliza-se do mesmo conceito de Promoção da Saúde preconizado na Carta de Ottawa, a qual menciona que promover a saúde fundamenta-se em proporcionar, às populações, alternativas necessárias para que tenham condições de melhorar sua saúde e exercer um maior controle sobre a mesma ${ }^{(17)}$. Nessa visão, a saúde é compreendida como a possibilidade da pessoa aproveitar a vida de maneira positiva, com a perspectiva de usar os recursos pessoais e sociais a seu alcance, indo além da capacidade física(18).

Com isso, salienta-se que a participação social e o desenvolvimento de habilidades pessoais são opções favoráveis à saúde dos idosos e encontram-se entre os campos de atuação da promoção da saúde. Assim, reforça-se a idéia que compreende o trabaIho voluntário como uma das formas de atuação social, servindo como uma alternativa para a promoção da saúde dos idosos, pois se acredita que o mesmo atue auxiliando na manutenção da saúde e na melhoria da qualidade de vida das pessoas nessa faixa etária. Em outros países, essa assertiva já encontra subsídios na literatura científica.

$\mathrm{Na}$ China, por exemplo, um estudo transversal realizado com 501 idosos aposentados (328 voluntários e 173 não voluntários), encontrou que os idosos voluntários reportaram melhor saúde física e satisfação com a vida e menor índice de depressão, comparados com os idosos que não realizavam trabalho voluntário - considerando $p<0,05(19)$.

Em Israel, um estudo longitudinal contemplando idosos a partir dos 75 anos, verificou que os que realizavam trabalho voluntário não se diferenciaram significativamente dos não-voluntários quanto ao número de patologias. Entretanto, os voluntários apresentaram menor déficit cognitivo, menos sintomas depressivos, maior número de relacionamentos interpessoais e avaliação mais positiva da vida ${ }^{(20)}$. Outras pesquisas realizadas recente- 
mente demonstram que o trabalho voluntário está associado com melhor satisfação pela vida, auto-estima positiva, melhor saúde física, auto-avaliação positiva da saúde, menor risco para mortalidade e tratamento positivo para depressão(4).

Essa relação entre trabalho voluntário e a melhoria das condições de saúde e da qualidade de vida reforça os pressupostos da Teoria da Atividade, que aponta a manutenção de atividades como um benefício e uma necessidade para a satisfação com a vida na velhice, enfatizando que a maioria dos idosos requer e deseja níveis elevados de atividade social. Considera que a pessoa que envelhece em boas condições é aquela que permanece ativa e que consegue resistir a vários obstáculos, em especial ao desengajamento social|(21-22).

No Brasil, há necessidade de estudos que investiguem os efeitos do voluntariado na saúde dos idosos que o realizam, pois pouco se publicou a respeito. Nessa perspectiva, reafirma-se a premência de despertar o interesse em investigar essa temática, a fim de fornecer subsídios que legitimem o trabalho voluntário como atividade que promova a saúde dos idosos brasileiros.

\section{CONSIDERAÇÕES FINAIS}

0 progressivo e rápido envelhecimento da população brasileira é um fenômeno que traz preocupação à sociedade e ao sistema de saúde, despreparado para atender as demandas geradas por um país envelhecido. Analisando as estimativas que apontam que em 2025 o país ocupará o sexto lugar entre as nações com maior número de idosos ${ }^{(1)}$, evidencia-se a importância de investigações que conduzam a novos rumos no sentido da promoção da saúde dos idosos.

Sabe-se que a cada ano que passa, as pessoas chegam à velhice com melhores condições de saúde e qualidade de vida, entretanto, muitas vezes essa situação não é mantida. Com a velhice, surge a aposentadoria e com esta, muitas vezes, o ostracismo e o isolamento social. A aposentadoria, no sistema capitalista, é vista como um período declinante da capacidade física. Dessa maneira, essa fase serve, muitas vezes, como um marco negativo, pois a sociedade rotula 0 aposentado como alguém inútil socialmente, excluindo-0 do mercado de trabalho, inclusive ${ }^{(23)}$. Pode-se considerar, ainda, que a aposentadoria e o excesso de tempo livre, para muitas pessoas, são situações que diminuem a satisfação pela vida e dispõem a saúde a uma maior vulnerabilidade ${ }^{(19)}$. Assim, para muitos idosos, o trabalho voluntário desponta como uma ferramenta que possibilita sentirem-se ativos e úteis socialmente, atuando como um mecanismo conservador da saúde e da qualidade de vida.

A crescente adesão das pessoas idosas ao voluntariado tem revelado que essa população, quando incentivada, se engaja em diversos trabalhos, independentemente de lu- cro financeiro(7). Há que se considerar, todavia, as dificuldades encontradas nos países em desenvolvimento, como o Brasil. Enquanto nos países desenvolvidos os voluntários, em geral, são pessoas com condições de ter suas necessidades básicas satisfeitas (alimentação, segurança, moradia, serviço de saúde e salário, entre outras), nos países em desenvolvimento essas garantias são privilégio de pequena parcela da população. Por esse motivo, muitos idosos são obrigados a terem, mesmo quando aposentados, novas funções remuneradas com vistas a garantirem sua sobrevivência ou completarem a renda familiar, criando, com isso, limitações no desenvolvimento e estimulação do trabalho voluntário ${ }^{(24)}$.

Características que influem no voluntariado, tais como classe social, experiências pessoais, cultura, escolaridade, filosofia e orientação religiosa, afetam tanto os voluntários dos países desenvolvidos quanto os pertencentes aos países em desenvolvimento, mas as desigualdades sociais típicas desses últimos podem acarretar dificuldades adicionais. Diante das carências da população dos países em desenvolvidos, a motivação e a organização do voluntariado pressupõe investimentos maiores do que nos países desenvolvidos, pois é necessário primeiramente dar respostas às necessidades dos próprios voluntários que, apesar da vontade, algumas vezes possuem grandes carências, muitas vezes não diferindo da clientela que ajudam a atender ${ }^{(24)}$.

Dessa maneira, faz-se necessário mencionar que não se deve intitular ou impor o voluntariado como uma solução para todas as pessoas. Considera-se esta atividade como uma alternativa eficiente para alguns idosos e não para todos, pois se admite a heterogeneidade e a diversidade entre as pessoas, que é ainda mais acentuada nos idosos.

Acredita-se que os profissionais de enfermagem podem atuar incentivando e despertando o pensamento crítico dos idosos, em todos os níveis de atenção à saúde, principalmente durante as consultas de enfermagem. Ao identificar idosos em situação de isolamento social ou com dificuldade para adaptação à aposentadoria, entre outras situações, o enfermeiro deve propor - levando-se em consideração as particularidades dos indivíduos - o trabalho voluntário como uma das alternativas para a inclusão social e uma opção para o tempo livre desses.

As instituições sociais e de saúde podem facilitar a prática do trabalho voluntário, permitindo o acesso a essa atividade, através da criação de espaço físico e ambiente favorável. Para isso, é necessária a parceria entre as instituições (privadas ou governamentais) e as ONGs voltadas para essa atividade, a fim de criarem-se ambientes propícios e acolhedores para a realização dessa prática. A experiência profissional permite dizer que em alguns hospitais essa iniciativa já é parcialmente adotada, onde é possível a atuação de voluntários ajudando às pessoas 
hospitalizadas. Contudo, ainda não se observa a participação dos profissionais de saúde nessa ação, tanto participando diretamente no voluntariado quanto incentivando para que os idosos nele atuem.

Sabe-se que esse investimento no voluntariado é um desafio aos profissionais, sobrecarregados por terem que enfrentar situações precárias do sistema de saúde. Entretanto, é fundamental a superação desses problemas, investindo-se em posturas criativas, inovadoras e diferenciadas, no intuito de encontrar novos horizontes para 0 atendimento à população.

\section{REFERÊNCIAS}

1. Organização das Nações Unidas (ONU). Plano de Ação Internacional para o Envelhecimento -2002. Brasília: Secretaria Especial dos Direitos Humanos; 2003.

2. Organização Mundial de Saúde (OMS). Organização PanAmericana de Saúde (OPAS). Envelhecimento ativo: uma política de saúde. Brasília; 2005.

3. Fischer LR, Mueller DP, Cooper PW. Older volunteers: a discussion of the Minnesota Senior Study. Gerontologist. 1991;31(2):183-94.

4. Wilson J. Volunteering. Annu Rev Sociol. 2000;26:215-40.

5. Penner LA. Dispositional and organizational influences on sustained volunteerism: an interactionist perspective. J Soc Issues. 2002;58(3):447-67.

6. Figueiredo NCM. Interfaces do trabalho voluntário na aposentadoria [dissertação]. Porto Alegre: Instituto de Psicologia, Universidade Federal do Rio Grande do Sul; 2005.

7. Lafin SHF, Souza SR, Barbosa C. Trabalho voluntário. In: Freitas EV, Py L, Cançado FAX, Doll J, Gorzoni ML, organizadores. Tratado de geriatria e gerontologia. $2 a$ ed. Rio de Janeiro: Guanabara Koogan; 2006. p. 1420-3.

8. Brasil. Lei n. 9.608, de 18 de fevereiro de 1998. Dispõe sobre o serviço voluntário e dá outras providências [legislação na Internet]. São Paulo: TRT/SP; 2003 [citado 2006 ago. 4]. Disponível em: http://www.trt02.gov.br/Geral/Tribunal2/Legis/Leis/9608_98.htm

9. Silva HABD. 0 voluntariado entre idosos no município de São Paulo [dissertação]. São Paulo: Faculdade de Saúde Pública, Universidade de São Paulo; 2003.

10. Frias SR. Aposentadoria, tempo livre, lazer e a descoberta do trabalho voluntário. In: Veras RP, oraganizador. Terceira idade: alternativas para uma sociedade em transição. Rio de Janeiro: UNATI; 1999. p. 183-9.

11. Souza JN, Chaves EC. 0 efeito do exercício de estimulação de memória em idosos saudáveis. Rev Esc Enferm USP. 2005;39(1):13-9.

12. Cerqueira ATAR, Oliveira NIL. Programa de apoio a cuidadores: uma ação terapêutica e preventiva na atenção à saúde dos idosos. Psicol USP. 2002;13(1):133-50.

13. Salas Junior AR, Gago JG, Mosca W. La salud y el bienestar de los adultos mayores. In: Briceño-Leon R, Minayo M CS, Coimbra Junior CEA, organizadores. Salud y equidad: una mirada desde las ciencias sociales. Rio de Janeiro: FIOCRUZ; 2000. p. 337-48.
Assim, reafirma-se a importância de se estimular (no sentido de despertar o interesse e não de impor condutas) e de se facilitar 0 acesso dos idosos à realização do trabalho voluntário. A própria OMS ratifica essa afirmativa, realçando que atitudes criadoras de ambientes de apoio e promotoras de opções saudáveis são fundamentais para políticas que almejam fornecer saúde e qualidade de vida aos ido$\mathrm{sos}^{(2)}$. Dessa forma, realça-se que é uma tarefa dos profissionais de saúde e da sociedade fomentar o prolongamento do período de vida ativa e saudável das pessoas, ao invés de, unicamente, se preocupar em acrescentar anos à vida.

14. Lehr U. A revolução da longevidade: impacto na sociedade, na família e no indivíduo. Estud Interdiscipl Envelhec. 1999;1(1):7-35.

15. Organización Panamericana de La Salud (OPAS). División de Promoción y Protección de la Salud. Salud de las Personas de Edad. Envejecimiento y salud: un cambio de paradigma. Rev Panam Salud Publica. 2000; 7(1):60-7.

16. Buss PM. Uma introdução ao conceito de promoção da saúde. In: Czeresnia D, Freitas CM, organizadores. Promoção da saúde: conceitos, reflexões e tendências. Rio de Janeiro: FIOCRUZ; 2003. p. 15-38.

17. Brasil. Ministério da Saúde. Secretaria de Políticas de Saúde. Projeto Promoção da Saúde. As cartas da promoção da saúde. Brasília; 2002. (Série B. Textos básicos em saúde).

18. Souza AC, Colomé ICS, Costa LED, Oliveira DLLC. A educação em saúde com grupos na comunidade: uma estratégia facilitadora da promoção da saúde. Rev Gaúcha Enferm. 2005;26(2):147-3.

19. Wu AMS, Tang CSK, Yan ECW. Post-retirement voluntary work and psychological functioning among older Chinese in Hong Kong. J Cross Cult Gerontol. 2005; 20(1):27-45.

20. Shmotkin D, Blumstein T, Modan B. Beyond keeping active: concomitants of being a volunteer in old-old age. Psychol Aging. 2003;18(3):602-7.

21. Eliopoulos C. Enfermagem gerontológica. 5a ed. Porto Alegre: Artmed; 2005.

22. Siqueira MEC. Teorias sociológicas do envelhecimento. In: Freitas EV, Py L, Néri AL, Cançado FAX, Gorzoni ML, Rocha SM, organizadores. Tratado de geriatria e gerontologia. Rio de Janeiro: Guanabara Koogan; 2002. p. 47-57.

23. Both TL. Jubilamento: 0 interdito de uma vida de trabaIho e suas repercussões na velhice [dissertação]. Porto Alegre: Instituto de Psicologia, Universidade Federal do Rio Grande do Sul; 2004.

24. Lafin SHF. As instituições comunitárias e o voluntariado idoso. In: Freitas EV, Py L, Néri AL, Cançado FAX, Gorzoni $M L$, Rocha SM, organizadores. Tratado de geriatria e gerontologia. Rio de Janeiro: Guanabara Koogan; 2002. p. $1048-50$. 